\title{
CAPACIDADE DE SUPORTE DE CARGA DE UM LATOSSOLO VERMELHO-AMARELO EM UMA LAVOURA CAFEEIRA ${ }^{(1)}$
}

\author{
Karina Marie Kamimura ${ }^{(2)}$, Moacir de Souza Dias Júnior ${ }^{(3)}$, Paulo Tácito Gontijo \\ Guimarães $^{(4)}$, Gérson Rodrigues dos Santos ${ }^{(5)} \&$ Marcelo Silva de Oliveira ${ }^{(6)}$
}

\begin{abstract}
RESUMO
O tráfego intensivo de máquinas agrícolas sobre solos cultivados com cafeeiros pode causar compactação excessiva, principalmente quando realizado sob condição inadequada de umidade, promovendo redução da produtividade das áreas sob intensa passagem de máquinas. Os objetivos deste trabalho foram: avaliar a degradação da estrutura do solo na linha de tráfego do trator (LTT) e na linha de tráfego da colhedora (LTC), por meio dos atributos físicos do solo e da capacidade de suporte de carga do solo em função do potencial matricial do solo; e calcular os potenciais matriciais críticos, para evitar a compactação adicional do solo pelo uso de um trator marca Massey Ferguson, modelo 275, e de uma colhedora Jacto KTR Advance em um Latossolo Vermelho-Amarelo textura muito argilosa. $\mathrm{O}$ estudo foi realizado na Fazenda da EPAMIG situada no município de PatrocínioMG. Para obtenção dos modelos de capacidade de suporte de carga $\left(\sigma_{p}\right)$ versus potencial matricial do solo, coletaram-se amostras indeformadas na LTT e LTC, nas camadas de 0-3, 10-13 e 25-28 cm, em cinco trincheiras, as quais foram submetidas ao ensaio de compressão uniaxial, em diferentes potenciais matriciais. Além disso, coletaram-se amostras indeformadas em seis trincheiras, para determinação dos atributos físicos do solo na LTT e LTC, nas camadas de 0-3, 10-13 e 25-28 cm. As operações realizadas com o trator degradaram mais a estrutura do solo do que as realizadas com a colhedora, pois na linha de tráfego do trator (LTT) a densidade do solo, a microporosidade e a pressão de
\end{abstract}

(1) Parte da Tese de doutorado do primeiro autor apresentada ao Programa de Pós-Graduação em Ciência do Solo do Departamento de Ciência do Solo da Universidade Federal de Lavras - DCS/UFLA. Recebido para publicação em 02 de maio de 2011 e aprovado em 06 de agosto de 2012.

(2) Doutoranda do Programa de Pós-Graduação em Ciência do Solo do DCS/UFLA, Campus Universitário, Caixa Postal 3037. CEP 37200-000 Lavras (MG). E-mail: karinamarie.kamimura@gmail.com

(3) Professor Associado do DCS/UFLA. Bolsista CNPq. Pesquisador Mineiro da FAPEMIG. E-mail: msouzadj@dcs.ufla.br

(4) Pesquisador do Centro Tecnológico do Sul de Minas, Empresa de Pesquisa Agropecuária de Minas Gerais (CTSM/EPAMIG), Campus da UFLA. E-mail: paulotgg@epamig.br

(5) Professor Adjunto, Departamento de Estatística, Universidade Federal de Viçosa, Av. P.H. Rolfs, s/n. CEP 36570-000 Viçosa (MG). E-mail: gerson.santos@ufv.br

(6) Professor Associado, Departamento de Ciências Exatas/UFLA. E-mail: marcelo.oliveira@dex.ufla.br 
preconsolidação foram maiores que as da linha de tráfego da colhedora. $\mathrm{O}$ potencial matricial crítico para a LTT foi de $-51 \mathrm{kPa}\left(0,36 \mathrm{~m}^{3} \mathrm{~m}^{-3}\right)$, e para a LTC, de $-291 \mathrm{kPa}\left(0,30 \mathrm{~m}^{3} \mathrm{~m}^{-3}\right)$.

Termos de indexação: compactação, pressão de preconsolidação, potencial matricial crítico.

\title{
SUMMARY: LOAD BEARING CAPACITY OF A RED-YELLOW LATOSOL IN A COFFEE PLANTATION
}

\begin{abstract}
The intensive agricultural traffic on soils under coffee can cause excessive compaction, mainly when vehicles pass under inadequate soil moisture conditions, promoting yield decreases. Our objectives were: a) to assess the degradation of soil structure in the tractor track (TT) and the harvester track (HT), based on soil physical properties and load bearing capacity as related to the soil matric potential in three layers, $b$ ) to calculate the critical matric potential to prevent additional soil compaction by a Massey Ferguson wheel tractor model 275 and a Jacto KTR Advance harvester on a clayey Red Latosol (Oxisol). The study was carried out at the Epamig Research Farm in Patrocinio, State of Minas Gerais, Brazil. Undisturbed soil samples were collected (layers 0-3, 1013 and $25-28 \mathrm{~cm}$ ) from five trenches. The models of soil bearing capacity $\left(\sigma_{p}\right)$ in function of soil matric potentials were determined in uniaxial compression tests with the undisturbed soil samples, at different soil matric potentials. In addition, soil samples were collected in six trenches (layers 0-3, 10-13 and 25-28 cm) for the determination of soil physical properties in the TT and HT. Transactions with the tractor degraded the soil structure more than operations with the harvester, since soil bulk density, microporosity and preconsolidation pressure were higher in the tractor track (TT) than in the harvester track $(H T)$. The critical matric potential of TT was $-51 \mathrm{kPa}\left(0.36 \mathrm{~m}^{3} \mathrm{~m}^{-3}\right)$ and the critical matric potential of $\mathrm{HT}-291 \mathrm{kPa}\left(0.30 \mathrm{~m}^{3} \mathrm{~m}^{-3}\right)$.
\end{abstract}

Index terms: compactation, pressure preconsolidation, matric potencials.

\section{INTRODUÇÃO}

O Brasil é uns dos principais produtores e exportadores de café do mundo, seguido por Vietnã, Colômbia e Indonésia. Minas Gerais é o Estado brasileiro que detém a maior área plantada com cafeeiros, com aproximadamente 1.000.751 ha, ou seja, $47,8 \%$ do total cultivado no Brasil, sendo grande parte em solos originalmente sob vegetação de cerrados. A produção nacional corresponde a 39,6 \% da produção mundial, podendo atingir 39 milhões de sacas de $60 \mathrm{~kg}$, dependendo do efeito da bienalidade (Agrianual, 2011).

O tráfego intensivo de máquinas agrícolas sobre solos cultivados com cafeeiros nas regiões do Triângulo Mineiro e do Alto Paranaíba, resultante principalmente do controle fitossanitário e manejo (Araújo-Júnior, 2011), tem comprometido suas propriedades físicas, como: porosidade total, macroporosidade, densidade do solo, retenção de água (Gontijo et al., 2008; Araújo-Júnior et al., 2008; Ajayi et al., 2009), condutividade hidráulica do solo e as taxas de infiltração de água (Timm et al., 2006) e pressão de preconsolidação (Santos et al., 2010; Araújo-
Júnior et al., 2011). Quando essas operações são realizadas em condição de maior conteúdo de água no solo, aumenta a probabilidade de dano à sua estrutura (Dias Júnior et al., 2007), em razão da baixa capacidade de suporte de carga.

Assim, o uso de máquinas agrícolas nas várias etapas do processo de produção, em condições inadequadas de umidade, tem sido o principal responsável por acarretar a degradação da estrutura do solo, diminuindo o seu potencial produtivo (Dias Junior, 2000; Araújo-Júnior et al., 2011; Severiano et al., 2011). Portanto, os diferentes manejos realizados nas lavouras cafeeiras têm promovido alterações na estrutura do solo, sobretudo nas entrelinhas da cultura, resultantes das frequentes operações de controle de plantas invasoras; essas alterações podem causar compactação do solo, com consequente redução na produtividade dos cafeeiros (Araújo-Júnior, 2011).

A compactação do solo compromete a sustentabilidade do sistema agrícola, alterando o desenvolvimento das plantas, diminuindo a aeração do solo, comprometendo a infiltração e tornando o solo propenso à erosão (Oliveira et al., 2003). Várias propriedades físicas solo, como resistência do solo à 
penetração (Tormena \& Roloff, 1996), densidade do solo e porosidade total, têm sido utilizadas como forma de identificar o problema da compactação. A pressão de preconsolidação recentemente vem destacando-se como uma propriedade eficaz de identificar e prevenir a compactação do solo (Dias Junior \& Pierce, 1996), pois é uma medida da capacidade de suporte de carga do solo, definida como a capacidade da estrutura do solo em resistir a tensões induzidas pelo tráfego de máquinas ou pisoteio animal, sem mudanças irreversíveis no arranjo tridimensional das partículas constituintes do solo (Alakukku et al., 2003), em uma determinada umidade ou potencial matricial.

A pressão de preconsolidação é obtida a partir da curva de compressão do solo (Dias Júnior \& Pierce, 1996; Silva et al., 2000; Imhoff et al., 2004; Keller et al., 2004). De acordo com esses autores, ela é definida como a máxima pressão que o solo sofreu no passado. Quando se aplicam pressões inferiores à pressão de preconsolidação, o solo sofre deformações pequenas, elásticas e reversíveis, ou seja, quando cessada a aplicação da pressão, o solo não apresentará compactação adicional. Por outro lado, quando são aplicadas pressões superiores à pressão de preconsolidação, o solo sofre deformações plásticas, as quais são irreversíveis; nesse caso, quando cessada a aplicação da pressão, o solo sofrerá compactação adicional.

Para que o solo não apresente compactação adicional e, consequentemente, não tenha a sua estrutura degradada, a pressão exercida pelos dispositivos de tração das máquinas e implementos agrícolas, assim como pelas patas dos animais, não deve ultrapassar a pressão de preconsolidação (Dias Júnior \& Pierce, 1996; Keller et al., 2004). Portanto, a pressão de preconsolidação é uma propriedade de grande utilidade na definição de práticas de manejo que visem à manutenção da qualidade física do solo.

O conhecimento da capacidade de retenção de água no solo torna-se fundamental em estudos de comportamento compressivo e trafegabilidade dos solos agrícolas, uma vez que, para uma mesma condição de manejo, o conteúdo de água no solo é o fator que governa a quantidade de deformação que poderá ocorrer (Imhoff et al., 2004; Severiano et al., 2009).

Neste estudo, buscou-se definir o potencial matricial crítico na linha de tráfego do trator e da colhedora em três camadas para que a compactação adicional fosse evitada. Lebert et al. (2007) sugerem que a distribuição das pressões aplicadas pelas máquinas agrícolas seja determinada em dois potenciais matriciais $(-6 \mathrm{kPa}$ e $33 \mathrm{kPa}$ ), que representam dois níveis de capacidade de campo e o maior risco de compactação durante as operações agrícolas mecanizadas.

$\mathrm{O}$ entendimento dos fatores que influenciam a capacidade de suporte de carga e o conhecimento dos níveis de pressão aplicados ao solo pelos equipamentos é essencial para a adequação do manejo do tráfego de máquinas em lavouras cafeeiras, a fim de manter a qualidade física do solo e a longevidade da lavoura cafeeira. Diante dessas considerações, este estudo foi realizado com os objetivos de: avaliar a degradação da estrutura do solo na linha de tráfego do trator (LTT) e na linha de tráfego da colhedora (LTC), por meio dos atributos físicos do solo e da capacidade de suporte de carga do solo em função do potencial matricial do solo; e calcular os potenciais matriciais críticos, para evitar a compactação adicional do solo pelo uso de um trator marca Massey Ferguson, modelo 275, e de uma colhedora Jacto KTR Advance em um Latossolo Vermelho-Amarelo, textura muito argilosa.

\section{MATERIAL E MÉTODOS}

O estudo foi realizado em uma lavoura de café (Coffea arabica L.), implantada em fevereiro de 1999, localizada no município de Patrocínio (MG). Antes da implantação da lavoura cafeeira, a área foi utilizada com pastagem. A área total do experimento é de $6.000 \mathrm{~m}^{2}$, com cafeeiros implantados no espaçamento de $3,80 \times 0,70 \mathrm{~m}$. A área encontra-se a $18^{\circ} 59^{\prime} 31^{\prime \prime} \mathrm{S}$ de latitude sul e a $46^{\circ} 59^{\prime} 31^{\prime \prime} \mathrm{W}$ de longitude a oeste de Greenwich, com altitude de $934 \mathrm{~m}$.

O município de Patrocínio está localizado numa área geologicamente complexa, conhecida como Arco da Canastra, que separa as bacias sedimentares dos rios São Francisco e Paraná. O clima da região, segundo a classificação de Köppen, é do tipo Aw, com duas estações bem definidas, inverno seco e verão chuvoso, com precipitação pluvial média anual de 1.620,1 mm (Silva \& Malvino, 2005).

A vegetação predominante da região é do tipo cerrado. O relevo é plano a suave ondulado, com declividade média de $3 \%$, sendo o solo classificado como um Latossolo Vermelho-Amarelo (Embrapa, 2006) textura muito argilosa (Quadro 1). Realizaramse as análises granulométricas via agitação lenta por um período de $16 \mathrm{~h}$, utilizado como dispersante físico, e o $\mathrm{NaOH} 0,1$ mol L-1 como dispersante químico (Embrapa, 1997). Foram determinados: areia (2,00-0,05 $\mathrm{mm})$, por tamisagem; o teor de argila $(<0,002 \mathrm{~mm})$, pelo método da pipeta; e de silte $(0,05$ $0,002 \mathrm{~mm})$, pela diferença entre o teor total da amostra de solo e os teores de areia e argila.

Quadro 1. Caracterização física de um Latossolo Vermelho-Amarelo, realizada nas camadas de 03, 10-13 e 25-28 cm. Média de quatro repetições

\begin{tabular}{cccccc}
\hline Camada & MO $^{(1)}$ & Dp $^{(2)}$ & Argila & Silte & Areia \\
\hline $\mathrm{cm}$ & dag kg $^{-1}$ & $\mathrm{Mg} \mathrm{m}^{-3}$ & & $\mathrm{~g} \mathrm{~kg}^{-1}$ & \\
\cline { 5 - 6 }-3 & 3,7 & 2,68 & 720 & 160 & 120 \\
$10-13$ & 2,6 & 2,70 & 640 & 230 & 130 \\
$25-28$ & 2,5 & 2,71 & 650 & 260 & 90 \\
\hline
\end{tabular}

${ }^{(1)} \mathrm{MO}$ : matéria orgânica do solo; ${ }^{(2)} \mathrm{Dp}$ : densidade de partículas. 
O controle de plantas invasoras foi feito com a aplicação de herbicida de pré-emergência com o ingrediente ativo oxyfluorfen. O controle fitossanitário foi realizado uma vez ao mês, utilizando pulverizador ARBUS 2000® com aproximadamente $2.600 \mathrm{~kg}$, quando completamente carregado. A adubação de cobertura, aplicada de 40 em 40 dias no período chuvoso, foi realizada utilizando-se uma adubadora modelo Fertinox; quando completamente carregada, tem capacidade para $600 \mathrm{~kg}$. Em todas as operações agrícolas foi utilizado um trator marca Massey Ferguson, modelo 275 (potência de $45 \mathrm{~kW}$ no motor $61 \mathrm{cv}$ ), com bitola de 1,015 m, pneus traseiros R1 18,4-30 com pressão de inflação de $96,5 \mathrm{kPa}$ e dianteiros R1 7,50-16 com pressão de inflação de $240 \mathrm{kPa}$, massa $3.900 \mathrm{~kg}$. A colheita foi realizada com uma colhedora Jacto KTR Advance com massa de $6.700 \mathrm{~kg}$ quando vazia, com graneleiro com capacidade de $150 \mathrm{~L}$, pneus - G2 10x24" 10 lonas, com pressão de $276 \mathrm{kPa}$ e bitola de $3,2 \mathrm{~m}$.

Para obtenção dos modelos de capacidade de suporte de carga (MCSC), foram coletadas em cinco trincheiras com dimensão de 4,5 x 2 m, nas camadas de 0-3, 1013 e $25-28 \mathrm{~cm}$, em duas regiões, na linha de tráfego do trator (LTT) e na linha de tráfego da colhedora (LTC), e sete potenciais matriciais, totalizando 210 amostras indeformadas, as quais foram saturadas e equilibradas nos potenciais matriciais $\left(-\psi_{\mathrm{m}}\right)$ de água de $2,6,10,33$, 100,500 e $1.500 \mathrm{kPa}$. Os potenciais matriciais de $-2 \mathrm{e}$ $-6 \mathrm{kPa}$ foram obtidos utilizando unidade de sucção (Grohmann, 1960); para obtenção dos demais potenciais, utilizou-se uma câmara de Richards (Klute, 1986). Após atingirem o equilíbrio, essas amostras foram submetidas ao ensaio de compressão uniaxial, usando um consolidômetro pneumático Terraload S450. Essas amostras foram coletadas em anéis metálicos de $2,54 \mathrm{~cm}$ de altura por $6,30 \mathrm{~cm}$ de diâmetro, com auxílio do amostrador tipo Uhland, e revestidas por filme plástico e parafina, com a finalidade de preservar a estrutura do solo até a realização das análises físicohídricas. Ressalta-se que as linhas de tráfego do trator e da colhedora não coincidem, pois os comprimentos das bitolas são diferentes.

Para os atributos físicos do solo, foram realizadas amostragens em seis trincheiras $(4,5 \times 2 \mathrm{~m})$ nas camadas de 0-3, 10-13 e 25-28 cm, na linha de tráfego do trator e da colhedora. As 36 amostras indeformadas foram coletadas, utilizando-se anéis metálicos de $2,54 \mathrm{~cm}$ de altura por $6,30 \mathrm{~cm}$ de diâmetro, com auxílio do amostrador tipo Uhland. As amostras foram revestidas por filme plástico e parafina, a fim de preservar a estrutura do solo até a realização das análises físicas. Os atributos físicos do solo usados foram: a densidade do solo (Ds), obtida pela relação entre a massa de solo seco e o volume do anel (Blake \& Hartge, 1986a); a densidade de partículas $(\mathrm{Dp})$, determinada pelo método do picnômetro, de acordo com Blake \& Hartge (1986b); a porosidade total (PT), determinada a partir da relação entre Ds e Dp, pela seguinte expressão: $\mathrm{PT}=[1-(\mathrm{Ds} / \mathrm{Dp})]$ (Embrapa, 1997); a macroporosidade do solo, obtida pela diferença entre PT e microporosidade, sendo esta considerada o conteúdo de água retido a potencial matricial de $-6 \mathrm{kPa}$ (Embrapa, 1997).

\section{Ensaio de compressão uniaxial}

As 210 amostras indeformadas (sete potenciais matriciais $\mathrm{x}$ três camadas $\mathrm{x}$ dois locais de amostragens $x$ cinco repetições) foram inicialmente saturadas por capilaridade e equilibradas em cada um dos seguintes potenciais matriciais $\left(-\psi_{\mathrm{m}}\right): 2,6,10,33$, 100,500 e $1.500 \mathrm{kPa}$; em seguida, elas foram usadas para obtenção da curva de retenção de água e dos modelos de capacidade de suporte de carga, sendo para isso submetidas ao ensaio de compressão uniaxial, usando consolidômetro pneumático Terraload S-450 (Durham Geo Enterprises, USA), conforme descrito por Dias Júnior (1994).

As amostras indeformadas foram mantidas dentro da célula de compressão e submetidas a pressões de $25,50,100,200,400,800$ e $1.600 \mathrm{kPa}$. Cada pressão foi aplicada até obter $90 \%$ da deformação máxima (Taylor, 1948), sendo então aplicadas sucessivamente as pressões crescentes, sem efetuar o descarregamento das pressões previamente aplicadas. A variação da deformação do solo foi monitorada por meio de um extensômetro.

As curvas de retenção de água (CRA) foram determinadas de acordo com van Genuchten (1980). Os conteúdos volumétricos de água $(\theta)$ foram ajustados de acordo com o potencial matricial $\left(\psi_{\mathrm{m}}\right)$, conforme a equação 1:

$$
\theta=\theta r+\left\{\theta s-\theta r /\left[1+\left(\alpha \psi_{m}\right)^{n}\right]^{m}\right\}
$$

em que $\theta_{\mathrm{s}}$ e $\theta_{\mathrm{r}}$ são o conteúdo de água na saturação e residual, respectivamente; e $\alpha, \mathrm{n}$ e m representam parâmetros empíricos de ajuste do modelo. Foi calculada também a água disponível, que foi obtida por diferença entre os teores de água do solo na capacidade de campo $\left(\theta_{\mathrm{cc}}\right)$ e no ponto de murcha permanente $\left(\theta_{\mathrm{PMP}}\right)$, conforme a equação 2 :

$$
\begin{aligned}
& {\left[\mathrm{AD}=\theta_{\mathrm{cc}}(-6 \mathrm{kPa})-\theta_{\mathrm{PMP}}(-1500 \mathrm{kPa})\right]} \\
& \text { (Reichardt, 1988; Oliveira et al., 2003). }
\end{aligned}
$$

\section{Determinação da pressão de preconsolidação}

A curva de compressão do solo foi obtida ajustandose graficamente, para cada amostra, o logaritmo da pressão aplicada (base 10) no eixo das abscissas e a densidade do solo no eixo das ordenadas. Os valores de pressão de preconsolidação $\left(\sigma_{\mathrm{p}}\right)$ foram determinados de acordo com Dias Junior \& Pierce (1995) e então ajustados em função do potencial matricial $\left(-\psi_{\mathrm{m}}\right)$, obtendo-se assim os modelos de capacidade de suporte de carga do solo.

A equação 3 permite calcular a $\sigma_{\mathrm{p}}$ em função do $-\psi_{\mathrm{m}}$ :

$$
\sigma_{\mathrm{p}}=\mathrm{a}+\mathrm{b} \ln \left(-\psi_{\mathrm{m}}\right)
$$


em que: $\sigma_{\mathrm{p}}=$ pressão de preconsolidação; $\mathrm{a}=$ coeficiente linear $\mathrm{b}=$ coeficiente angular; $-\psi_{\mathrm{m}}=$ potencial matricial do solo.

\section{Comparação dos modelos de capacidade de suporte de carga}

As análises de regressão dos modelos de capacidade de suporte de carga foram realizadas por meio do software Sigma Plot 8.0 (2002). As comparações dos modelos da capacidade de suporte de carga foram feitas de acordo com procedimento descrito por Snedecor \& Cochran (1989), o qual testa a homogeneidade dos dados (F) e a significância dos coeficientes lineares (a) e angulares (b) das equações linearizadas (Equação 3).

Equações de regressões lineares $\sigma_{\mathrm{p}}=\mathrm{a}+\mathrm{b} \ln \left(-\psi_{\mathrm{m}}\right)$ homogêneas entre si e coeficientes das regressões não significativos foram agrupados, sendo então ajustado um único modelo, considerando todos os valores de $\sigma_{\mathrm{p}} \mathrm{e}-\psi_{\mathrm{m}}$.

Os valores referentes a porosidade total, macroporosidade, microporosidade e densidade do solo foram submetidos a análises exploratórias, para obtenção da distribuição de frequência e normalidade dos dados pelo teste W (Shapiro \& Wilk, 1965). O delineamento experimental utilizado foi inteiramente casualizado, em esquema fatorial $2 \times 3$, sendo dois locais de tráfego (LTT e LTC) e três camadas (0-3, 10-13 e 25$28 \mathrm{~cm}$ ). Nas situações em que ocorreu normalidade dos dados, procedeu-se à análise de variância e à comparação das médias, utilizando o teste de Scott-Knott a $5 \%$, pelo programa estatístico Sisvar (Ferreira, 2000).

\section{RESULTADOS E DISCUSSÃO}

Na LTT, a porosidade total (PT) foi maior na camada de 25-28 cm, em comparação com as de 0-3 e $10-13 \mathrm{~cm}$, que não diferiram estatisticamente entre si, indicando que o tráfego alterou a PT do solo até a camada de 10-13 cm. O mesmo ocorreu para a LTC. A LTT apresentou menores valores de PT para todas as camadas analisadas, quando comparada com a LTC (Quadro 2).

De acordo com Kiehl (1979), um solo em condições ideais deve apresentar um terço da PT formada por macroporos e os dois terços restantes por microporos, estabelecendo uma relação macroporos/microporos igual a $0,5 . \mathrm{Na}$ LTC, a relação macroporos/microporos foi de 0,5 , enquanto na LTT essa relação foi de 0,30 , indicando que houve degradação na estrutura do solo, corroborando assim os resultados obtidos por Gontijo et al. (2008), os quais verificaram relação macroporos/ microporos de 0,5 na linha de tráfego e na projeção da saia do cafeeiro.

A macroporosidade na LTT, nas camadas de 0-3 e 10-13 cm, não diferiram estatisticamente entre si, apresentando menores valores em relação à camada de $25-28 \mathrm{~cm}$. Na LTC, verificou-se que a camada de
25-28 cm apresentou maior valor de macroporosidade, quando comparada às camadas $0-3$ e $10-13 \mathrm{~cm}$, que não diferiram estatisticamente entre si (Quadro 2).

Houve redução média de $36,4 \%$ de macroporos na LTT em relação à LTC. Segundo Gupta \& Allmaras (1987) e Kiehl (1979), o sistema radicular da maioria das plantas cresce satisfatoriamente quando a percentagem de macroporos está acima de $0,10 \mathrm{~m}^{3} \mathrm{~m}^{-3}$. Essa condição ideal foi verificada na LTC nas camadas de 0-3 e $10-13 \mathrm{~cm}$, cujo valor foi de $0,20 \mathrm{~m}^{3} \mathrm{~m}^{-3}$, e na camada de $25-28 \mathrm{~cm}$, com $0,26 \mathrm{~m}^{3} \mathrm{~m}^{-3}$; já na LTT, nas camadas $0-3$ e 10-13 cm verificou-se o valor de $0,12 \mathrm{~m}^{3} \mathrm{~m}^{-3}$ de macroporos, próximo ao citado limite crítico. A redução dos macroporos deve-se à destruição da estrutura do solo, que reduz o volume dos poros de maior tamanho, devido à carga excessiva aplicada ao solo pelas operações mecanizadas, principalmente na linha de tráfego (Gontijo et al., 2008).

$\mathrm{Na}$ LTT, a camada de $10-13 \mathrm{~cm}$ apresentou maior valor de microporos, quando comparada às de 0-3 e 25$28 \mathrm{~cm}$, que não diferiram estatisticamente entre si. Na LTC, as camadas de 0-3 e 10-13 cm não diferiram estatisticamente entre si, apresentando maiores valores de microporosidade em comparação com a de $25-28 \mathrm{~cm}$. Quanto à microporosidade, verificou-se aumento médio de $8,7 \%$ de microporos na LTT, quando comparada à LTC (Quadro 2). Valores próximos foram obtidos por Gontijo et al. (2008), que constataram aumento médio de $10 \%$ de microporos na linha de tráfego, quando comparada a entrelinha num Latossolo Vermelho distrófico típico sob lavoura cafeeira.

Quadro 2. Porosidade total, macroporosidade, microporosidade e densidade do solo, obtidas na linha de tráfego do trator e colhedora em três camadas, num Latossolo Vermelho-Amarelo

\begin{tabular}{|c|c|c|c|}
\hline \multirow[b]{2}{*}{ Tratamento } & \multicolumn{3}{|c|}{ Camada $(\mathrm{cm})$} \\
\hline & $0-3$ & $10-13$ & $25-28$ \\
\hline & \multicolumn{3}{|c|}{ Porosidade total ${ }^{(1)}, \mathrm{m}^{3} \mathrm{~m}^{-3}$} \\
\hline LTT & $0,58 \mathrm{aA}$ & $0,59 \mathrm{aA}$ & $0,62 \mathrm{bA}$ \\
\hline \multirow[t]{2}{*}{ LTC } & $0,63 \mathrm{aB}$ & $0,63 \mathrm{aB}$ & $0,65 \mathrm{bB}$ \\
\hline & \multicolumn{3}{|c|}{ Macroporosidade ${ }^{(1)}, \mathrm{m}^{3} \mathrm{~m}^{-3}$} \\
\hline LTT & $0,12 \mathrm{aA}$ & $0,12 \mathrm{aA}$ & $0,18 \mathrm{bA}$ \\
\hline \multirow[t]{2}{*}{ LTC } & $0,20 \mathrm{aB}$ & $0,20 \mathrm{aB}$ & $0,26 \mathrm{bB}$ \\
\hline & \multicolumn{3}{|c|}{ Microporosidade ${ }^{(1)}, \mathrm{m}^{3} \mathrm{~m}^{-3}$} \\
\hline LTT & $0,45 \mathrm{aA}$ & $0,48 \mathrm{bA}$ & $0,44 \mathrm{aA}$ \\
\hline \multirow[t]{2}{*}{ LTC } & $0,43 \mathrm{aB}$ & $0,43 \mathrm{aB}$ & $0,39 \mathrm{bB}$ \\
\hline & \multicolumn{3}{|c|}{ Densidade do solo ${ }^{(1)}, \mathrm{Mg} \mathrm{m}^{-3}$} \\
\hline LTT & $1,13 \mathrm{aA}$ & $1,1 \mathrm{aA}$ & $1,03 \mathrm{bA}$ \\
\hline LTC & $0,98 \mathrm{aB}$ & $1,01 \mathrm{aB}$ & $0,95 \mathrm{bB}$ \\
\hline
\end{tabular}

(1) Média de seis repetições. Médias seguidas da mesma letra, minúscula nas linhas e maiúscula nas colunas, dentro de cada atributo, não diferem entre si a $5 \%$ pelo teste de Scott-knott. LTT - linha de tráfego do trator; LTC - linha de tráfego da colhedora. 
A densidade do solo (Ds) foi maior para as camadas de 0-3 e 10-13 cm na LTT, que diferiram estatisticamente da camada de $25-28 \mathrm{~cm}$; o mesmo ocorreu na LTC. Araújo-Júnior et al. (2011) verificaram que o valor da Ds crítico para o Latossolo Vermelho distroférrico foi de $1,2 \mathrm{Mg} \mathrm{m}^{-3}$. Os valores de DS desse estudo variaram de 0,95 a $1,13 \mathrm{Mg} \mathrm{m}^{-3}$, estando abaixo do valor considerado crítico obtido por Araújo-Júnior et al. (2011).

A redução na PT condicionou maiores valores de Ds na LTT, onde se constatou aumento médio de $11 \%$ na Ds quando comparada à LTC (Quadro 2). Analisando os resultados da PT, macroporosidade e Ds, observa-se que a operação crítica em relação à compactação é a realizada com o trator que apesar de ter menor massa (trator + pulverizador $=6.500 \mathrm{~kg}$ ou trator + adubadora $=4.500 \mathrm{~kg})$ do que a colhedora quando cheia $(6.850$ $\mathrm{kg})$. Essa maior compactação do solo deve-se à frequência de operações e à época do ano (estação chuvosa), induzindo maior compactação no solo do que a colhedora, que realiza a colheita na estação seca.

As curvas de retenção de água no solo foram influenciadas pelo tráfego de máquinas (Figura 1). Na LTT, verificou-se que a água disponível nas camadas de 0-3, 10-13 e $25-28 \mathrm{~cm}$ foi de $0,12,0,13$ e $0,14 \mathrm{~m}^{3} \mathrm{~m}^{-3}$, respectivamente. Já na LTC a água disponível nas camadas de $0-3,10-13$ e $25-28 \mathrm{~cm}$ foi de $0,14,0,15$ e $0,14 \mathrm{~m}^{3} \mathrm{~m}^{-3}$, respectivamente. Na camada de $0-3 \mathrm{~cm}$ na LTC, verificou-se que a água disponível foi $17 \%$ maior em relação à LTT. Já na camada de $10-13 \mathrm{~cm}$ a água disponível na LTC foi $15 \%$ maior em relação à LTT; na camada de $25-28 \mathrm{~cm}$ o valor de água disponível foi igual para a LTT e LTC. Segundo Beutler

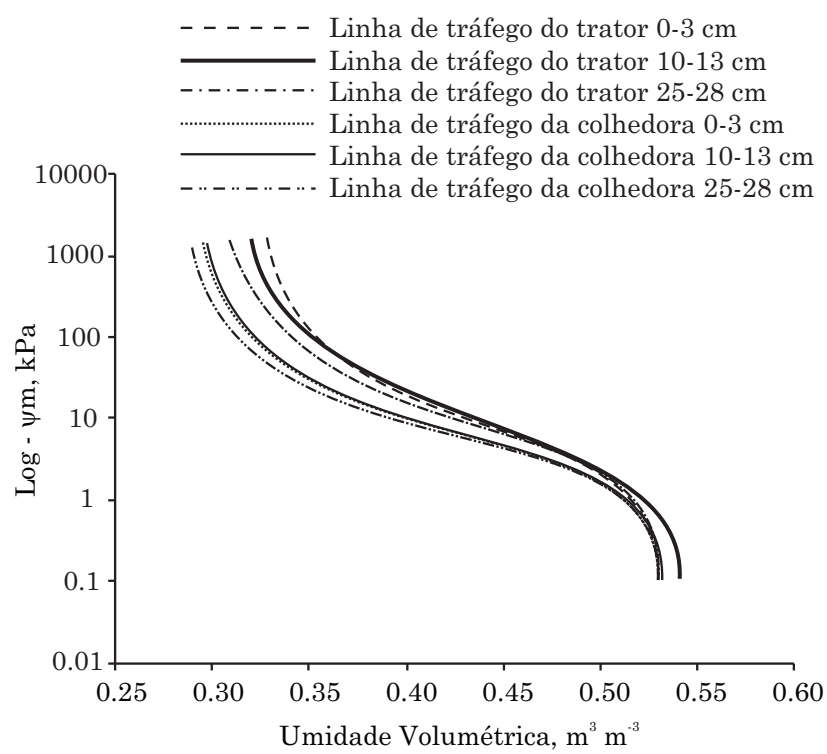

Figura 1. Curva de retenção de água do solo na linha de tráfego do trator e colhedora em três camadas, num Latossolo Vermelho-Amarelo cultivado com café. et al. (2002), o conteúdo de água retido no solo é característica específica de cada solo, sendo resultado da ação conjunta e complexa de vários fatores; ele depende do teor e mineralogia da fração argila, da Ds e do teor de matéria orgânica.

Os modelos de capacidade de suporte de carga (MCSC) na LTT nas camadas de 0-3 e 10-13 cm não se diferenciaram estatisticamente, sendo, portanto, uma nova equação ajustada a todos os valores de $\sigma_{\mathrm{p}} \mathrm{e}$ $-\psi_{\mathrm{m}}$, obtendo-se assim um único MCSC para estas camadas. Esse modelo foi diferente estatisticamente do modelo da camada de 25-28 cm (Quadro 3). Essa camada foi a mais suscetível à compactação. $\mathrm{O}$ mesmo ocorreu para a LTC (Quadro 3 e Figura 2).

Quadro 3. Comparação entre as regressões lineares dos modelos de capacidade de suporte de carga. $\sigma_{\mathrm{p}}=\mathrm{a}+\mathbf{b} \ln \left(-\psi_{\mathrm{m}}\right)$, de acordo com Snedecor \& Cochran (1989), para o Latossolo VermelhoAmarelo cultivado com café

\begin{tabular}{|c|c|c|c|}
\hline Linha de tráfego e camada & $\mathbf{F}$ & $\begin{array}{c}\text { Coeficiente } \\
\text { linear, a }\end{array}$ & $\begin{array}{c}\text { Coeficiente } \\
\text { angular, b }\end{array}$ \\
\hline Trator ${ }_{0-3} \mathrm{x}_{10-13 \mathrm{~cm}}$ & $\mathrm{H}$ & ns & ns \\
\hline Trator $_{0-3}$ e $10-13 \mathrm{~cm} \times$ Trator $_{25-28 \mathrm{~cm}}$ & $\mathrm{NH}$ & * & * \\
\hline Colhedora $_{0-3 \mathrm{~cm}} \times$ Colhedora $_{10-13 \mathrm{~cm}}$ & $\mathrm{H}$ & ns & ns \\
\hline Colhedora $_{0-3}$ e $10-13 \mathrm{~cm} \times$ Colhedora $_{25-28 \mathrm{~cm}}$ & $\mathrm{H}$ & * & $*$ \\
\hline Trator $_{0-3}$ e $10-13 \mathrm{~cm} \times$ Colhedora $_{0-3}$ e $10-13 \mathrm{~cm}$ & $\mathrm{H}$ & ns & * \\
\hline Trator $_{25-28 \mathrm{~cm}} \times$ Colhedora $_{25-28 \mathrm{~cm}}$ & $\mathrm{H}$ & $*$ & * \\
\hline
\end{tabular}

NH: não homogêneo; H: homogêneo; ns: não significativo; *: significativo a $5 \%$.

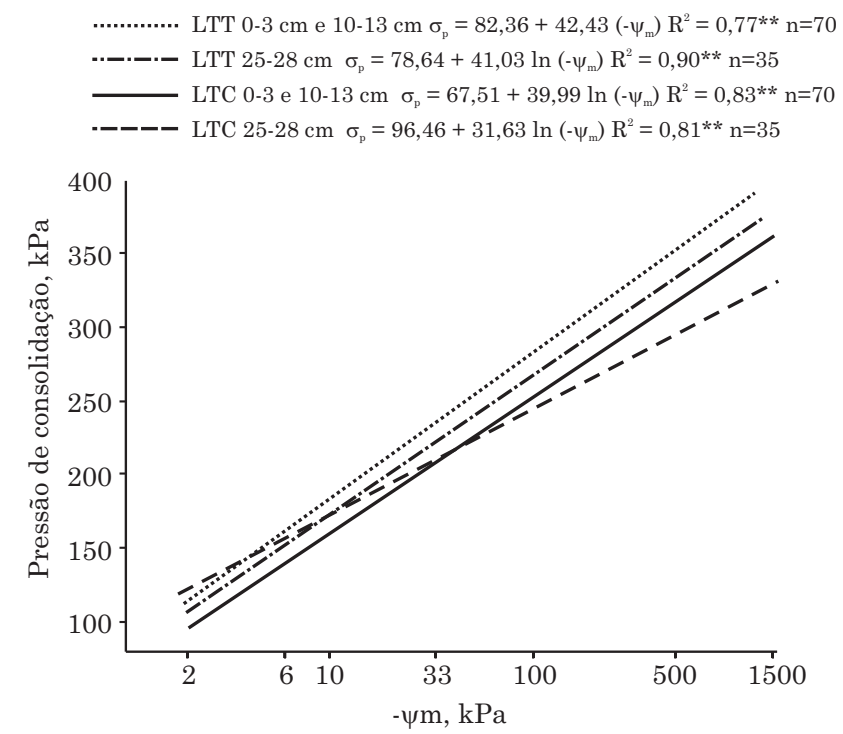

Figura 2. Modelo de capacidade de suporte de carga em função do potencial matricial $\left(-\psi_{\mathrm{m}}\right)$ em um Latossolo Vermelho-Amarelo (LVA) para a linha de tráfego do trator (LTT) e linha de tráfego da colhedora (LTC), em três camadas sob a cultura do café. 
Quando se compararam os MCSC na LTT e LTC, verificou-se diferença estatística para todas as camadas, indicando diferentes capacidades de suporte de carga (CSC) para o solo em estudo; portanto, os modelos não foram agrupados (Quadro 4). A LTT em todos os potenciais apresentou maiores CSC do que a LTC para todas as camadas, indicando que o trator degrada mais a estrutura do solo, o que é evidenciado pelas maiores CSC do solo (Figura 2).

A maior CSC na LTT (Figura 2) proporcionou maiores valores de microporosidade e DS e menores valores de PT e macroporosidade. Solos com maior capacidade de suporte de carga podem ser benéficos à trafegabilidade de máquinas para o manejo da lavoura cafeeira; entretanto, isso está ligado à maior compactação do solo (Araújo-Júnior et al., 2008), o que pode alterar o crescimento do sistema radicular e o transporte de água e de assimilados das raízes para a parte aérea (Rena \& Guimarães, 2000).

$\mathrm{Na}$ LTT, onde ocorre intenso tráfego do trator, foram verificados maiores valores médios da $\sigma_{\mathrm{p}}$, o que está relacionado à elevada frequência de tráfego durante o ano. Segundo Silva et al. (2006), no manejo de lavouras cafeeiras, em média são realizadas entre 12 e 17 operações mecanizadas anuais. Já a colhedora apresentou menores valores de $\sigma_{p}$, o que pode estar relacionado à menor frequência dessa operação e ao fato de que a colheita é realizada na época seca, quando o solo apresenta maior CSC, minimizando a possibilidade de ocorrer compactação do solo.

Apesar de Horn et al. (2007) sugerirem que os valores das pressões dinâmicas sejam até cinco vezes maiores do que os da pressão estática, a seguir é apresentada uma proposta dos valores dos potenciais matriciais e umidade volumétrica, em que o trator e a colhedora podem trafegar sem promover compactação adicional, considerando a pressão estática, devido à falta de informações sobre as pressões dinâmicas aplicadas pelos equipamentos usados nas lavouras cafeeiras.

De acordo com o manual do fabricante, os rodados do trator aplicam uma pressão estática máxima de $240 \mathrm{kPa}$; em razão disso, o tráfego deve ser realizado, nas camadas de 0-3 e 10-13 cm, em potencial matricial maior do que de - $41 \mathrm{kPa}$, que corresponde à umidade volumétrica de $0,38 \mathrm{~m}^{3} \mathrm{~m}^{-3}$ (Figura 3a). Na camada de $25-28 \mathrm{~cm}$, o tráfego deve ser realizado em potenciais

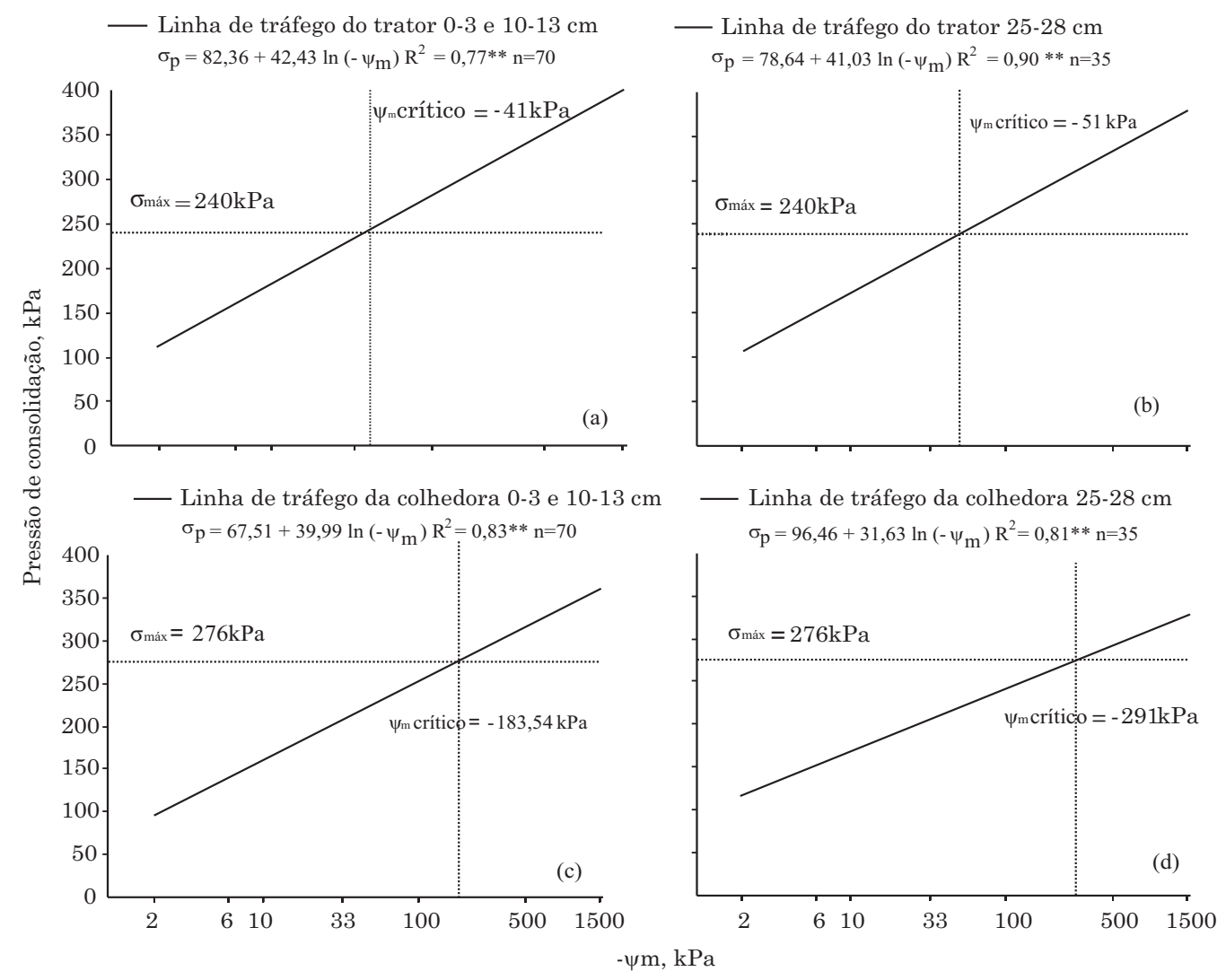

Figura 3. Modelo de capacidade de suporte de carga em função do potencial matricial ( $\left.-\psi_{m}\right)$ em um Latossolo Vermelho-Amarelo (LVA) para a linha de tráfego do trator (LTT) e linha de tráfego da colhedora (LTC), em três camadas de estudo sob a cultura do café. A linha pontilhada horizontal representa a pressão máxima aplicada pelo rodado do trator e colhedora, e a linha pontilhada vertical representa o potencial matricial crítico. 
matriciais maiores do que $-51 \mathrm{kPa}$, que corresponde à umidade volumétrica de $0,36 \mathrm{~m}^{3} \mathrm{~m}^{-3}$ (Figura $3 b$ ).

Araújo-Júnior et al. (2011) determinaram as umidades volumétricas críticas no centro das entrelinhas de cafeeiros para um trator com pressão máxima de $220 \mathrm{kPa}$ sob diferentes sistemas de manejo de plantas invasoras, em um Latossolo Vermelho distroférrico. Esses autores verificaram que o menor valor de umidade crítica foi de $0,27 \mathrm{~m}^{3} \mathrm{~m}^{-3}$ para o solo sob o manejo sem capina na camada de $0-3 \mathrm{~cm}$, e o maior valor foi de $0,48 \mathrm{~m}^{3} \mathrm{~m}^{-3}$ para o solo sob o manejo herbicida de pré-emergência na camada de $0-3 \mathrm{~cm}$.

Como consta no manual do fabricante, os rodados da colhedora aplicam uma pressão estática máxima de $276 \mathrm{kPa}$; em razão disso, o tráfego deve ser realizado nas camadas de $0-3$ e $10-13 \mathrm{~cm}$ nos potenciais matriciais maiores do que $-183,54 \mathrm{kPa}$, correspondendo à umidade volumétrica de $0,31 \mathrm{~m}^{3} \mathrm{~m}^{-3}$ (Figura 3c). Na camada de 25-28 cm, o tráfego deve ser realizado no potencial matricial maior que $-291 \mathrm{kPa}$, que corresponde à umidade volumétrica de $0,30 \mathrm{~m}^{3} \mathrm{~m}^{-3}$ (Figura 3d). No entanto, por questão de segurança, devem-se considerar as camadas menos resistentes à compactação para que o tráfego de máquinas seja realizado sem promover compactação do solo, estabelecendo, portanto, como limites os valores de $-51 \mathrm{kPa}\left(0,36 \mathrm{~m}^{3} \mathrm{~m}^{-3}\right)$ e $-291 \mathrm{kPa}$ $\left(0,30 \mathrm{~m}^{3} \mathrm{~m}^{-3}\right)$ para a LTT e LTC, respectivamente.

\section{CONCLUSÕES}

1. As operações realizadas com o trator degradaram mais a estrutura do solo do que aquelas realizadas com a colhedora, pois na linha de tráfego do trator (LTT) a densidade do solo, a microporosidade e a pressão de preconsolidação foram maiores que na linha de tráfego da colhedora (LTC).

2. O potencial matricial crítico para a LTT foi de $-51 \mathrm{kPa}\left(0,36 \mathrm{~m}^{3} \mathrm{~m}^{-3}\right)$, e para a LTC, de $-291 \mathrm{kPa}$ $\left(0,30 \mathrm{~m}^{3} \mathrm{~m}^{-3}\right)$.

\section{AGRADECIMENTOS}

Os autores agradecem ao Consórcio Brasileiro de Pesquisa e Desenvolvimento do Café - CBP\&D/Café o apoio financeiro para o desenvolvimento do presente estudo; aos funcionários da Fazenda Experimental da EPAMIG, em Patrocínio (MG). O primeiro autor agradece à CAPES a concessão da bolsa de doutorado.

\section{LITERATURA CITADA}

ANUÁRIO DA AGRICULTURA BRASILEIRA - AGRIANUAL. Mercados e perspectivas do café. São Paulo, FNP, 2011. p.192-210.
AJAYI, A.E.; DIAS JUNIOR, M.S.; CURI, N.; ARAÚJOJÚNIOR, C.F.; SOUZA, T.T.T. \& INDA JUNIOR, A.V. Strength attributes and compaction susceptibility of Brasilian Latosols. Soil Tillage Res., 105:122-127, 2009.

ALAKUKKU, L.; WEISSKOPF, P.; CHAMEN, W.C.T.; TIJINK, F.G.J.; van der LINDEN, J.P.; PIRES, S.; SOMMER, C. \& SPOOR, G. Prevention strategies for field traffic induced subsoil compaction: a review: part I - machine/soil interactions. Soil Tillage Res., 73:145-160, 2003.

ARAÚJO-JÚNIOR, C.F.; DIAS JÚNIOR, M.S.; GUIMARÃES, P.T.G. \& ALCÂNTARA, E.N. Capacidade de suporte de carga e umidade crítica de um Latossolo induzida por diferentes manejos. R. Bras. Ci. Solo, 35:115-131, 2011.

ARAÚJO-JÚNIOR, C.F.; DIAS JUNIOR, M.S.; GUIMARÃES, P.T.G. \& PIRES, B.S. Resistência a compactação de um Latossolo cultivado com cafeeiro, sob diferentes sistemas de plantas invasoras. R. Bras. Ci. Solo, 32:25-32, 2008.

BLAKE, G.R. \& HARTGE, K.H. Bulk density. In: KLUTE, A., ed. Methods of soil analysis. 2.ed. Madison, ASA/SSSA, 1986a. p.363-375. (Agronomy Monograph, 9)

BLAKE, G.R. \& HARTGE, K.H. Particle density. In: KLUTE, A., ed. Methods of soil analysis. 2.ed. Madison, ASA/SSSA, 1986b. p.377-382. (Agronomy Monograph, 9)

BEUTLER, A.N.; CENTURION, J.F.; SOUZA, Z.M.; ANDRIOLI, I. \& ROQUE, C.G. Retenção de água em dois tipos de Latossolos sob diferentes usos. R. Bras. Ci. Solo, 26:829-834, 2002.

DIAS JÚNIOR, M.S. Compactação do solo. In: NOVAIS, R.F.; ALVAREZ V., H.V. \& SCHAEFER, C.E.G.R. Tópicos em Ciência do solo. Viçosa, MG, Sociedade Brasileira de Ciência do Solo, 2000. v.1. p.55-94.

DIAS JÚNIOR, M.S. Compression of three soils under longterm tillage and wheel traffic. East Lansing, Michigan State University, 1994. 114p. (Tese de Doutorado)

DIAS JÚNIOR, M.S.; FONSECA, S.; ARAÚJO-JÚNIOR, C.F. \& SILVA, A.R. Soil compaction due to Forest harvest operations. Pesq. Agropec. Bras., 42:257-264, 2007.

DIAS JÚNIOR, M.S. \& PIERCE, F.J. A simple procedure for estimating preconsolidation pressure from soil compression curves. Soil Technol., 8:139-151, 1995.

DIAS JÚNIOR, M.S. \& PIERCE, F.J. O processo de compactação do solo e sua modelagem. R. Bras. Ci. Solo, 20:175-182, 1996.

EMPRESA BRASILEIRA DE PESQUISA AGROPECUARIA EMBRAPA. Centro Nacional de Pesquisas de Solos. Manual de métodos de análises de solo. 2.ed. Brasília, Produção de Informação, 1997. 212p.

EMPRESA BRASILEIRA DE PESQUISA AGROPECUÁRIA EMBRAPA. Sistema brasileiro de classificação de solos. 2.ed. Rio de Janeiro, 2006. 306p.

FERREIRA, D.F. Análises estatísticas por meio do SISVAR para Windows 4. 0. In: REUNIÃO ANUAL DA REGIÃO BRASILEIRA DA SOCIEDADE INTERNACIONAL DE BIOMETRIA, 45., São Carlos, 2000. Anais... São Carlos, Universidade Federal de São Carlos, 2000. p.255-258. 
van GENUCHTEN, M.T. A closed-form equation for predicting hydraulic conductivity of unsaturated soils. Soil Sci. Soc. Am. J., 44:892-898, 1980.

GONTIJO, I.; DIAS JUNIOR, M.S.; GUIMARÃES, P.T.G. \& ARAÚJO-JÚNIOR, C.F. Atributos físico-hídricos de um Latossolo de cerrado em diferentes posições de amostragem na lavoura cafeeira. R. Bras. Ci. Solo, 32:2227$2234,2008$.

GROHMANN, F. Distribuição e tamanho de poros em três tipos de solos do estado de São Paulo. Bragantia, 21:319$328,1960$.

GUPTA, S.C. \& ALLMARAS, R.R. Models to asses the susceptibility of soils to excessive compaction. Adv. Soil Sci., 6:5-10, 1987.

HORN, R.; VOSSBRINK, J.; PETH, S. \& BECKER, S. Impact of forest model vehicles on soil physical properties. For. Ecol. Manage., 248:56-63, 2007.

IMHOFF, S.; SILVA, A.P. \& FALLOW, D. Susceptibility to compaction, load support capacity, and soil compressibility of Hapludox. Soil Sci. Soc. Am. J., 68:391-411, 2004.

KELLER, T.; ARVIDSSON, J.; DAWIDOWSKI, J.B. \& KOOLEN, A.J. Soil precompression stress - II. A comparison of different compaction tests and stressdisplacement behaviour of the soil during wheeling. Soil Tillage Res., 77:97-108, 2004.

KIEHL, E.L. Manual de edafologia. São Paulo, Ceres, 1979. 262p.

KLUTE, A. Laboratory measurement of hydraulic conductivity of satured soils. Madison, American Society of Agronomy, 1986. p.253-261. (Monograph, 9)

LEBERT, M.; BÖKEN, H. \& GLANTE, F. Soil compaction indicators for assessment of harmful changes to the soil in the context of the German Federal Soil Protection Acta J. Environ. Manage., 82:388-397, 2007.

OLIVEIRA, G.C.; DIAS JÚNIOR, M.S.; CURI, N. \& RESCK, D.V.S. Compressibilidade de um Latossolo Vermelho argiloso de acordo com a tensão de água no solo, uso e manejo. R. Bras. Ci. Solo, 27:773-781, 2003.

REICHARDT, K. Capacidade de campo. R. Bras. Ci. Solo, 12:211-216, 1988.

RENA, A.B. \& GUIMARAES, P.T.G. Sistema radicular do cafeeiro: Estrutura, distribuição, atividade e fatores que o influenciam. Belo Horizonte, EPAMIG, 2000. 80p. (Documentos, 37)
SANTOS, G.A.; DIAS JUNIOR, M.S.; GUIMARÃES, P.T.G. \& PAIS, P.S.M. Suscetibilidade a compactação de um Latossolo Vermelho-Amarelo submetido a diferentes métodos de controle de plantas invasoras, na cultura cafeeira. Coffee Sci., 5:123-136, 2010.

SEVERIANO, E.C.; OLIVEIRA, G.C.; CURI, N. \& DIAS JUNIOR, M.S. Potencial de uso e qualidade estrutural de dois solos cultivados com cana-de-açúcar em Goianésia (GO). R. Bras. Ci. Solo, 1:159-168, 2009.

SEVERIANO, E.C.; OLIVEIRA, G.C.; DIAS JÚNIOR, M.S.; COSTA, K.A.P.; BENITES, V.M. \& FERREIRA FILHO, S.M. Structural changes in Latosols of the cerrado region: ii - soil compressive behavior and modeling of additional compaction. R. Bras. Ci. Solo, 35:783-791, 2011.

SHAPIRO, S.S. \& WILK, M.B. An analysis of variance test for normality: Complete samples. Biometrika, 52:591-611, 1965.

SIGMA PLOT. Scientific Graphing Software. Versão 8.0. Software. San Rafael, Jandel Corporation, 2002.

SILVA, A.R.; DIAS JUNIOR, M.S.; GUIMARÃES, P.T.G. \& ARAUJO JUNIOR, C.F. Modelagem da capacidade de suporte de carga e quantificação dos efeitos das operações mecanizadas em um Latossolo Amarelo cultivado com cafeeiros. R. Bras. Ci. Solo, 30:207-216, 2006.

SILVA, E.M. \& MALVINO, S.S.A.B. Análise climática do município de Patrocínio (MG). Caminhos Geogr., 10:93108, 2005.

SILVA, V.R.; REINERT, D.J. \& REICHERT, J.M. Suscetibilidade à compactação de um Latossolo VermelhoEscuro e de um Podzólico Vermelho-Amarelo. R. Bras. Ci. Solo, 24:239-249, 2000.

SNEDECOR, G.W. \& COCHRAN, W.G. Statistical methods. 8.ed. Ames, Iowa State University Press, 1989.

TAYLOR, D.W. Fundamentals of soil mechanics. New York, John Wiley, 1948. 770p.

TIMM, L.C.; PIRES, L.F.; ROVERATTI, R.; ARTHUR, R.C.J.; REICHARDT, K.; OLIVEIRA, J.C.M. \& BACCHI, O.O.S. Field spatial and temporal patterns of soil water content and bulk density changes. Sci. Agric., 63:5564, 2006.

TORMENA, C.A. \& ROLOFF, G. Dinâmica da resistência à penetração de um solo sob plantio direto. R. Bras. Ci. Solo, 20:333-339, 1996 